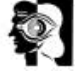

columns but I would suggest that they have less relevance to audit and appraisal than research. Indeed, it is all too often forgotten that clinical audit uses standard research methods and will generate unreliable results unless carefully designed (Lawrie \& Sandercock, 2004).

One of the main problems with the higher trainees research day as it currently operates is that it is often inadequately organised and supervised. Most trainees would gain a lot more from a 4- or 6-month slot in full-time research as part of a research group, and this would constitute only a ninth or a sixth of a 3-year training programme. Such attachments could be allocated to those who requested them, as other training posts are at present. Overall, this would probably increase the numbers of psychiatrists with research skills; this would be important not only for audit but also for the promotion of research of direct clinical relevance. If our practice is to be influenced by more than politics and fashion, we need more research in psychiatry rather than less.

LAWRIE, S. M., SANDERCOCK, P. (2004) Epidemiology and research methods. In Companion to Psychiatric Studies (7th edn) (eds E. C. Johnstone, D. G. C. Owens, S. M. Lawrie, et al) pp. 65-184. Edinburgh: Churchill Livingstone.

Stephen M. Lawrie Sackler Senior Clinical Research Fellow, Royal Edinburgh Hospital, Edinburgh EH10 5HF

\section{Research for the sake of research}

I wholeheartedly agree with the views expressed by Nicola Phillips in her letter 'Who wants to be a specialist registrar?' (Psychiatric Bulletin, March 2005, 29, 115) One of the biggest worries one has when applying for specialist registrar posts is the absence of research experience on one's CV.

Research is clearly very important for the advancement of psychiatry or for any other specialty for that matter. It is also important that trainees be encouraged to do research work. But does every single trainee have to do research work? Not everybody has the same aptitudes and interests; research for the sake of research is not very helpful. Some trainees are more interested in being good clinicians or have other special interests. For example, a special interest in psychotherapy should be given the same weight age as one in research.

It is also time to consider the research day that all registrars are given. It might not be the most effective use of time for someone who is not interested in research. As much as science needs good research, patients need good clinicians and psychiatry needs professionals with different interests.
Pavan Chahl Staff Grade in General Adult Psychiatry, Hellesdon Hospital, Norwich

\section{A case for the 4-month SHO post?}

With Part II of the MRCPsych examination rapidly approaching, I wonder how far the College has gone in considering reducing the length of training posts to 4 months from the current 6

Several medical rotations have already embraced this approach in order to provide breadth of training within the limited time available. There seem to be several reasons why this approach might also be suited to psychiatry.

First, having completed the required posts for examination entry, including 6 months in neurology, I am soon to enter my 4th year as a senior house officer (SHO) and would still benefit from experience in forensic, psychiatric intensive treatment unit, perinatal and specialist addiction service roles. Four-month posts would allow all of this experience to be gained within 3 years, and allow time to be spent in research prior to entry into higher specialist training.

Second, competitive posts along with those required for examination entry can at times be in short supply and there will be a continued need for suitable placements to be found for general practitioner trainees, pre-registration house officers in their 2 nd foundation year and perhaps in the future SHOs in medicine/neurology. Shorter posts should reconcile some of these competing demands if staffed appropriately while at the same time: (a) increasing exposure to psychiatry among other medical professionals, and access to medicine/neurology among psychiatric trainees; (b) decreasing stigma via familiarity; (c) facilitating recruitment; and (d) reducing some of the historical barriers between psychiatry and the rest of medicine that seem so much of an anachronism today.

Brent Elliott Senior House Officer, A \& E Liaison and Crisis Intervention Service, Royal London Hospital, Whitechapel E1 1BB, e-mail: brentelliot@aol.com

\section{Moving consultant post}

Dr Smithies writes about her experience in moving consultant post (Psychiatric Bulletin, February 2005, 29, 65-66). We compared the characteristics of consultants who remained in post (stills) with those who moved to a new post (movers) using two surveys of workload and stress in consultant old age psychiatrists (Jolley \& Benbow, 1997: Benbow \& Jolley, 2002). Of those who contributed to both surveys, one-quarter changed post over 4 years. Movers did not differ significantly from stills in relation to age, gender, marital status or work pattern. Individua doctors described similar stress levels in both surveys, suggesting that stress profiles remain stable. Movers were slightly younger than stills, and more often came from small teams, rather than working alone or in a larger team (but these findings were not statistically significant). Measures of stress in the second survey did not differentiate between the groups.

The mobility of consultant psychiatrists is an important feature of National Health Service practice. Moving is not, however, associated with an abnormal stress profile, or a change in an individual's perceived level of stress.

A mobile workforce brings with it advantages and disadvantages. Bringing new ideas and approaches from one culture to another is enlivening and stimulating. It avoids the risk of staff losing enthusiasm through boredom and sameness. For patients and carers, it reduces the risk that institutionalisation will mask, conceal, excuse or condone poor or exploitative behaviour. However, too much change can be counterproductive: promoting uncertainty and undermining confidence, and reducing the efficiency derived from established interpersonal links.

BENBOW, S. M. \& JOLLEY, D. J. (2002) Burnout and stress among old age psychiatrists. International Journal of Geriatric Psychiatry, 17,710-714.

JOLLEY, D. J. \& BENBOW, S. M. (1997) The everyday work of geriatric psychiatrists. International Journal of Geriatric Psychiatry, 12, 109-113.

Susan M. Benbow Consultant Old Age Psychiatrist, Wolverhampton City Primary Care Trust and Professor of Mental Health and Ageing, Wolverhampton University, West MidlandsWV4 5HN, e-mail: susan. benbow@wlv.ac.uk, David J. Jolley Director of Dementia Plus West Midlands, Wolverhampton

\section{The objective structured clinical examination}

The case for and against the objective structured clinical examination (OSCE) has generated many interesting letters in the Bulletin recently. The unequivocal and emphatic response by Mortimer \& Lunn (Psychiatric Bulletin, December 2004, 28, 458 ) is welcome. It is clearly 'here to stay' (apology unnecessary, in my opinion)

The pros - examination of a broad range of scenarios, reducing the luck factor - outweigh, I feel, concerns about limited time and the fostering of 'fast psychiatry' (Yak et al, Psychiatric Bulletin, July 2004, 28, 265-266). It is a useful addition to the examination format. However, concerns about the challenge of handling the long case format deserve a response. 